\title{
Research Article \\ Design of Special Impacting Filter for Multicarrier ABPSK System
}

\author{
Zhimin Chen and Lenan Wu \\ School of Information Science \& Engineering, Southeast University, 2 Sipailou, Nanjing 210096, China \\ Correspondence should be addressed to Zhimin Chen; chenzhimin_118@163.com
}

Received 14 January 2013; Accepted 17 March 2013

Academic Editor: Yudong Zhang

Copyright ( 2013 Z. Chen and L. Wu. This is an open access article distributed under the Creative Commons Attribution License, which permits unrestricted use, distribution, and reproduction in any medium, provided the original work is properly cited.

\begin{abstract}
A rather intuitive technique known as pole-zero placement is introduced to illustrate the frequency response of the special impacting filters (SIFs) with a pair of conjugate zero-poles and deduce the equation of the pole radii. Based on that, the paper proposes an iterative scheme to derive the parameters of the cascade notch filter. The cost function is determined by the cascading notch filter's influence on impacting filters, converting the cost function's least square problem to a filter parameters' standard quadratic programming problem. Finally, a cascading notch SIF (CNSIF) designed to demodulate the ABPSK signals is realized.
\end{abstract}

\section{Introduction}

As we know, spectrum is an unrenewable resource, and the new wireless communication systems are occupying more and more transmission bandwidth. So many corporations and research institutions try to find the optimal method to utilize the spectrum. Against this background, the ultranarrow band (UNB) technology was proposed by doctor Walker. Up until now, various modulation methods have been successively proposed with the progress of UNB development, for example, variable phase shifting keying (VPSK), enhanced VPSK, very minimum shifting keying (VMSK), pulse position phase reversal keying (3PRK), missing cycle modulation (MCM), suppressed cycle modulation (SCM), and minimum sideband modulation (MSB), and so forth. The key of the demodulation is the "zero group delay" filter. The "zero group delay" means the rise time of the filter is very short and the phase changes are preserved after the "zero group delay" filter. The core unit is a quartz crystal which is too unreliable, instable, and inflexible to be massproduced [1-3]. Wu et al. analyzed the feasibility of the UNB and proposed a high-efficiency modulation called extended BPSK (EBPSK) $[4,5]$. In 2009, they proposed continuous phase EBPSK (CP-EBPSK) based on the EBPSK [6], further tightening and reducing the EPBSK power spectrum sideband. Subsequently, they imported the pseudorandom sequence and modified spectrum parameter to improve the CP-EBPSK, that is, modified CP-EBPSK (MCP-EBPSK). All of the above techniques have the same characteristic, that is "asymmetric." They are uniformly referred to as asymmetric binary phase shift keying (ABPSK). ABPSK could increase the transport bitrate and the spectrum utilization efficiency with a more narrow band [7]. The modulation of the ABPSK signal relies on the special impacting filter (SIF), which could convert phase changes to amplitude impacts. Details of the SIF will be given in Section 3 .

In recent years, multicarrier (MC) scheme has been recognized as a potential candidate for the physical layer of the wireless communication systems which could not only gain spectral efficiency but also lower out-of-band radiation. The first candidate of MC schemes is orthogonal frequencydivision multiplexing (OFDM) [8]; however, a number of shortcomings of OFDM in the MC systems have been noted as well, such as large side lobes of the frequency response of the filters that characterize the subcarrier channels, high peak-to-average power ratio (PAPR), and sensitivity to carrier frequency offset. Compared with OFDM, the ABPSK allows a high level of subchannel spectral containment as Figure 1 shows. So we propose that the ABSPK technique can be used in MC schemes. 


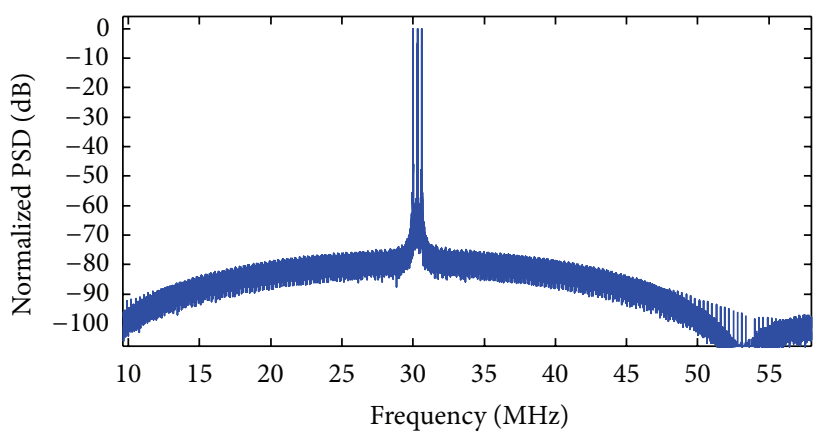

_ Power spectrum of three-subcarrier 1/4MCP-EBPSK signal

FIGURE 1: Power spectrum of 1/4MCP-EBPSK signal with three subcarriers.

\section{Uniform Formula of ABPSK}

Denote the following.

(1) Data bit duration $T$, it lasts $N \geq 1$ carrier wave period, that is, $T=N / f_{c}$.

(2) Phase modulation duration $\tau$, it lasts $K$ carrier wave period, that is, $\tau=K / f_{c}$ and $K \leq N$.

(3) $g_{0}$ and $g_{1}$ are modulation waveforms corresponding to bit " 0 " and bit " 1 ", respectively.

Then the uniform formula of ABPSK is defined as

$$
\begin{gathered}
g_{0}(t)=A \sin 2 \pi f_{c} t, \quad 0 \leq t<T, \\
g_{1}(t)= \begin{cases}B \sin \left(2 \pi f_{c} t \pm \sigma\right), & 0 \leq t<\tau, \quad 0 \leq \Delta \leq 1, \quad 0 \leq \eta \leq 1, \\
A \sin 2 \pi f_{c} t, & \tau \leq t<T .\end{cases}
\end{gathered}
$$

When the phase changes abruptly, $\sigma \in[0, \pi]$; when phase changes continuously, $\sigma= \pm \Delta \sin \left(\eta * 2 \pi f_{c} t\right)$. The modulated waveform has the following features.

(1) Data bit " 0 " lasts $N$ carrier cycles in $T$, the carrier cycle is $1 / f_{c}$ and the amplitude is $A$.

(2) Data bit " 1 " consists of $K$ carrier cycles with an initial phase $\sigma$, amplitude $B$ and $N-K$ carrier cycles with the same phase as bit " 0 ", and amplitude $A$.

(3) If there are no phase changes or amplitude changes, the waveforms are normal sine wave. When $\sigma \in$ $[0, \pi]$, it is the EBPSK; when $\sigma= \pm \Delta \sin \left(\eta * 2 \pi f_{c} t\right)$, according to the sign of $\Delta$ and value of $\eta$, it is the CPEBPSK and MCP-EBPSK, respectively.

(4) When $B=0, \sigma \in[0, \pi]$, (1) is MCM as has been mentioned in [9]; when $B=A$, (1) denote the following.

(a) When $\sigma=\pi, \tau=T$, it represents the classical BPSK; when $\tau=T / 4$, it represents the 3PRK in [9].

(b) When $\sigma=\pi / 2, \tau=T / 4$, it represents 3PSK in [9].

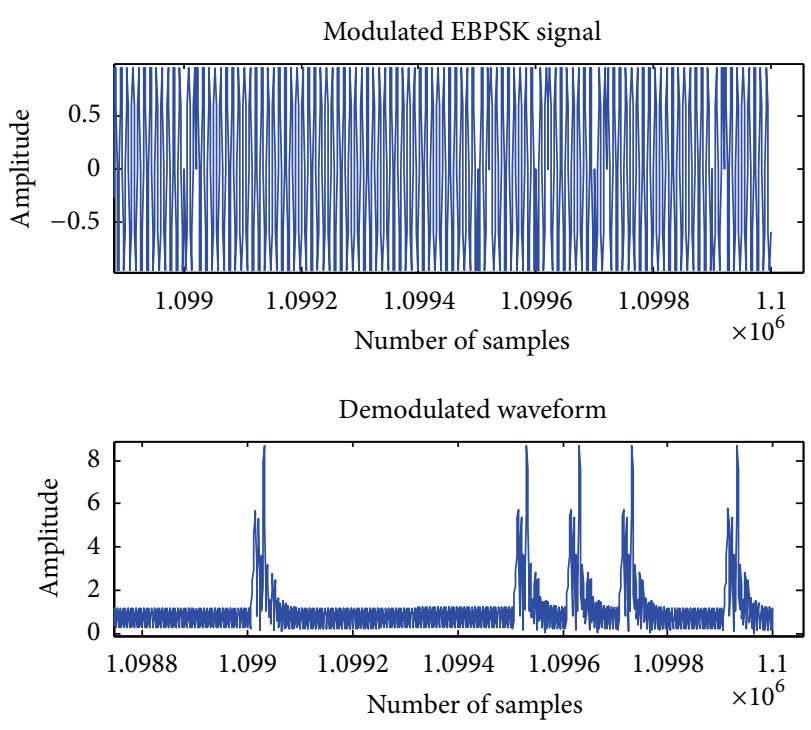

FIGURE 2: Modulated and demodulated waveform of EBPSK.

(5) The modulation degrees differ according to the phase change $S$ and modulate duration. The phase changes could control the bandwidth: when the phase vanishingly changes, the spectrum is the narrowest, and when the changes are $\pi$, the spectrum is the widest; Denote $\tau / T=K / N$ as "modulated duty cycle."

According to (1), choosing a proper value of $\Delta, \eta, \sigma, \tau$ and using a better shaping-filter could tighten the bandwidth of the modulated signal. We call the signal defined by (1) the uniformly asymmetric binary phase shift keying (ABPSK).

\section{Special Impacting Filter (SIF)}

The demodulation of AEBPSK signal based on the special digital impacting filter is in $[10,11]$. The SIF is a kind of digital IIR filters; when working on the proper frequency range, it can convert the phase changes to amplitude impacting. The places where phase is unchanged have much lower impacting than the changed places and most noise is removed at the same time (see Figure 2). Based on the output response, direct amplitude detection can be used for the symbol judgment in intermediate frequency instead of down-conversion to baseband. The filtering mechanism in that filter offers different band to signal and noise, which is discussed in $[10,11]$ in detail.

Different from normal multicarrier transmission system, the ABPSK subcarriers need not guard interval or satisfy the orthogonal condition. Every subchannel set differs from the others. A narrow pass band of the SIF is used to separate the subcarriers. In order to eliminate the adjacent channel interference, we need to set the notch frequency of the cascade notch filter the same as other carriers frequency to suppress other subchannels' interference and the output demodulation results [10]. The tiny phase change of the ABPSK makes the demodulation performance closely related to the sampling rate. Only by setting the proper parameter 


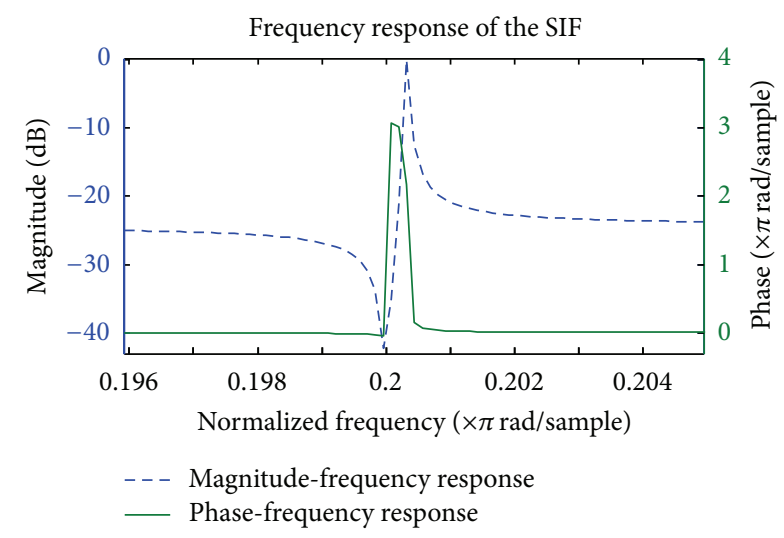

FIGURE 3: Frequency response of the SIF.

of the filter could it reflect the signal change. Based on the above condition, the parameters of the SIF bank correspond with each subcarrier's sampling rate, respectively.

\section{Design of Cascade Notch SIF (CNSIF)}

The key to demodulate the multicarrier ABPSK signal is the design of the receive filter bank, that is, cascade notch filter based on the SIF. Each subchannel regards the other subchannels as a sinusoidal interference, the notch filter cancelling the interference and SIF demodulating the corresponding signal. Many methods have been mentioned in $[10,11]$ for the SIF design and performance analysis, but the SIF pole radius is specified by the designer arbitrarily. Here we present a new approach to the numerical solution of the SIF as well as use an iterative scheme to gain the pole angle of the notch filter that fulfills the cost function.

4.1. Calculation of the SIF Pole Radii. Consider an SIF with one pair of conjugate zero-poles whose transfer function is

$$
H(z)=\frac{\left(1-r_{z} e^{j \omega_{c-\varepsilon}} z^{-1}\right)\left(1-r_{z} e^{-j \omega_{c-\varepsilon}} z^{-1}\right)}{\left(1-r_{p} e^{j \omega_{c+\mu}} z^{-1}\right)\left(1-r_{p} e^{j \omega_{c+\mu}} z^{-1}\right)} .
$$

Different from the conventional IIR filters, the zero angles are less than the corresponding carrier frequency and are less than the pole angles. Place the zeros on the unit circle with angle shift $\nu$, that is, $r_{z}=1, \omega_{z}=\omega_{c}-v$; the poles are close to the unit circle and are near to zeros with angle shift $\mu$, that is, $0 \ll r_{p}<1, \omega_{p}=\omega_{c}+\mu$. Generally the carrier frequency is situated in the maximum slope of the magnitude response. Figure 3 shows the amplitude-frequency response and phasefrequency response of the demodulation impacting filter with one pair of conjugate zero-poles, the normalized carrier frequency is $0.2 \pi \mathrm{rad} / \mathrm{sample}$; it has narrow notch-frequencyselecting performance near the carrier frequency.

According to (2), we can evaluate the frequency response with graphical method (see Figure 4). In practice the poles and zeros are even much closer than Figure 4; the poles are

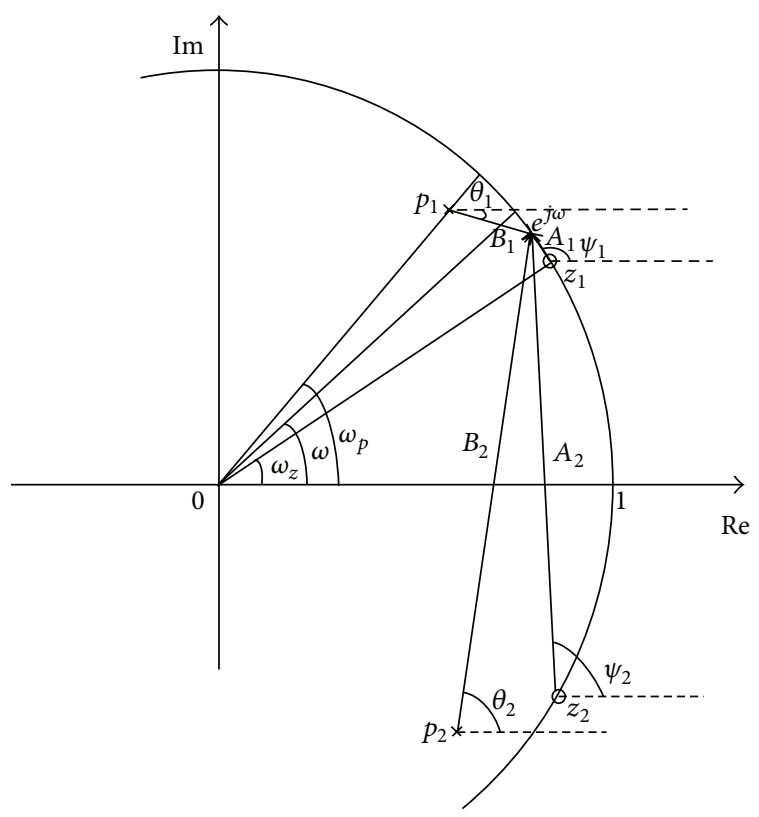

FIGURE 4: Schematic of zero-pole relative position.

even much closer to the unit circle. In the $z$-plane, the zeropole vectors shift on the unit circle $e^{j \omega}$.

$z_{1}, z_{2}$ are zeros lying on the unit circle; $p_{1}, p_{2}$ are poles near the unit circle and zeros. $A_{1}, \psi_{1}, A_{2}, \psi_{2}, B_{1}, \theta_{1}, B_{2}, \theta_{2}$ represent the module and angle of $z_{1}, z_{2}, p_{1}, p_{2}$, respectively. For the periodic frequency characteristic of the digital filters, we could just discuss the situation of $0<\omega<\omega_{s} / 2$, that is, $0 \sim \pi$.

From Figure 4, the frequency magnitude response of (2) can be rewritten as

$$
\left|H\left(e^{j \omega}\right)\right|=\frac{A_{1} \cdot A_{2}}{B_{1} \cdot B_{2}} .
$$

Because the zeros and poles are close to the unit circle and the distances from the conjugate pole and zero to $e^{j \omega}$ are nearly equal when they shift in the region of $0<\omega<\omega_{z}$ and $\omega_{p}<\omega<\omega_{s} / 2$; that is, $A_{1} \approx B_{1}, A_{2} \approx B_{2}$. So

$$
\left|H\left(e^{j \omega}\right)\right|=1, \quad 0<\omega<\omega_{z} \cup \omega_{p}<\omega<\frac{\omega_{s}}{2} .
$$

But when they shift in the region of $\omega_{z}<\omega<\omega_{p}$, the distance $A_{1}, B_{1}$ is much smaller than the distance $A_{2}, B_{2}$; we may state that $A_{2} \approx B_{2}$. The frequency magnitude response can be written as

$$
\left|H\left(e^{j \omega}\right)\right|=\frac{A_{1}}{B_{1}}, \quad \omega_{z}<\omega<\omega_{p} .
$$

Enlarge the region of $\omega_{z}<\omega<\omega_{p}$, the relative position between zero-pole vector and $e^{j \omega}$ depicted in Figure 5, here the influence of the complex conjugate zero-pole pair will be negligible. 


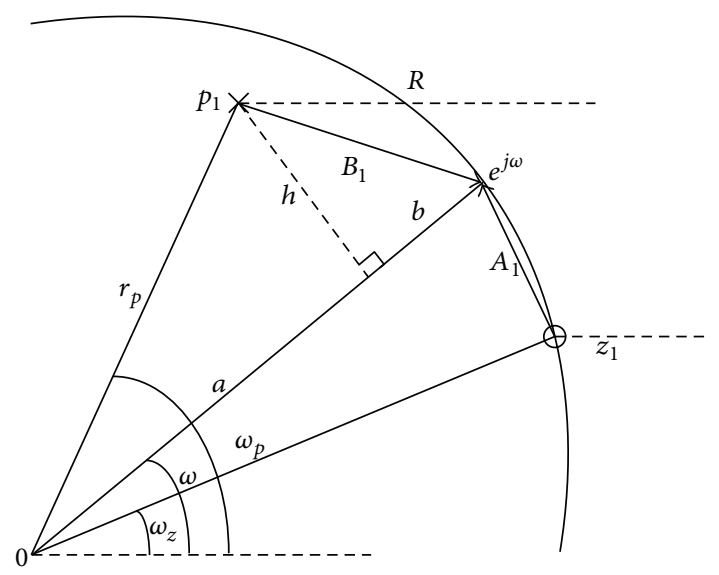

FIGURE 5: Schematic of the enlarged relative position between zeropole.

With the Pythagorean theorem, we obtain two equations:

$$
\begin{gathered}
A_{1}=2 \sin \left(\frac{\omega-\omega_{z}}{2}\right), \\
B_{1}=\sqrt{h^{2}+b^{2}}=\sqrt{r_{p}^{2}-a^{2}+b^{2}},
\end{gathered}
$$

where $r_{p}$ is the module of pole $p_{1}$.

Substituting $a=\sqrt{r_{p}^{2}-r_{p}^{2} \sin ^{2}\left(\omega_{p}-\omega\right)}, a+b=1$, into (7) leads to

$$
B_{1}=\sqrt{r_{p}^{2}-2 r_{p} \cos \left(\omega_{p}-\omega\right)+1}
$$

So the frequency magnitude response in the region of $\omega_{z}<\omega<\omega_{p}$ can be written as

$$
\left|H\left(e^{j \omega}\right)\right|=\frac{2 \sin \left[\left(\omega-\omega_{z}\right) / 2\right]}{\sqrt{r_{p}^{2}-2 r_{p} \cos \left(\omega_{p}-\omega\right)+1}}, \quad \omega_{z}<\omega<\omega_{p} .
$$

According to Moorer's bandwidth definition [12], if the resonance gain over the $\mathrm{DC}$ gain exceeds $6 \mathrm{~dB}$ in absolute value, the frequencies at $3 \mathrm{~dB}$ below/above the peak/notch is the band edges, that is

$$
\left|H\left(e^{j\left(\omega_{c}-B_{w} / 2\right)}\right)\right|=\frac{1}{\sqrt{2}} \cdot\left|H\left(e^{j \omega_{c}}\right)\right|
$$

where $B_{w}$ is the bandwidth of the SIF; (10) can be expressed as

$$
\frac{\sin \left[\left(\omega_{p}-\omega_{z}\right) / 2\right]}{\sin \left[\left(\omega_{p}-B_{w} / 2-\omega_{z}\right) / 2\right]} \cdot \frac{\sqrt{r_{p}^{2}-2 r_{p} \cos \left(B_{w} / 2\right)+1}}{1-r_{p}}=\sqrt{2}
$$

To ensure the impacting effect, the pole and zero angle is specified by the designer. When the bandwidth $B_{w}$ is known, the results of the following two equations are constants, respectively, that is

$$
\begin{gathered}
\omega_{p}-\omega_{z}=\phi, \\
\frac{2\left[1-\cos \left(\phi-B_{w} / 2\right)\right]}{1-\cos \phi}=M .
\end{gathered}
$$

Substituting (12) into (11), we can readily obtain

$r_{p}$

$$
=\frac{M-\cos \left(B_{w} / 2\right) \pm \sqrt{\cos ^{2}\left(B_{w} / 2\right)-2 M \cos \left(B_{w} / 2\right)+2 M-1}}{M-1} .
$$

The poles and zeros are very close to each other, as a consequence, $\phi$ and $B_{w}$ will be relatively small. To avoid $r_{p}>1$, we abandon the adding situation. After some further simplification. The final result is

$$
=\frac{M-\cos \left(B_{w} / 2\right)-\sqrt{\cos ^{2}\left(B_{w} / 2\right)-2 M \cos \left(B_{w} / 2\right)+2 M-1}}{M-1} .
$$

Equation (14) shows that the pole radii of SIF can be given in a quantitative way instead of arbitrary setting.

The procedure of the one-pair conjugate zero-poles SIF design could ensure the impacting effect, and we know that if the zeros and poles are far away from the unit circle, they will only affect the amplitude of the frequency response, but not the shape. From this standpoint, we can add more conjugate poles to tighten the bandwidth of the SIF and gain higher impacting effect. Generally we can add two conjugate poles whose angles are equal to the initial poles and are far away from the unit circle.

4.2. The Cascade Notch SIF (CNSIF). The multicarrier ABPSK scheme uses the CNSIF banks to separate and demodulate each subcarrier [13]. The key of the CNSIF is the narrow notch-frequency-selecting performance. When the overlapping signals pass the filter banks, the primary signal is enhanced and the noises or interferences are attenuated. Here the SIF enhances the primary signal and the notch filter decreases the disturbing signal. Let us start with the design of the notch filter in details.

Without loss of generality, we assume that the notch filter has $k$ notch frequencies; the transfer function is given by

$$
H_{1}(z)=\frac{B(z)}{A(z)}=\frac{\prod_{i=1}^{k}\left(1-2 \cos \left(\omega_{N i}\right) z^{-1}+z^{-2}\right)}{\prod_{i=1}^{k}\left(1-2 r_{i} \cos \left(\omega_{p i}\right) z^{-1}+r_{i}{ }^{2} z^{-2}\right)},
$$

where $r_{i}$ is the pole radius. For stability and according to system requirements, the values of $\omega_{N i}$ and $r_{i}$ are given by the designer $\left(0 \leq r_{i}<1\right)$. Meanwhile the zeros are constrained to 
locate on the unit circle at the notch frequencies $\omega_{N i}$, so the problem of designing a notch filter reduces to find the optimal value of $\omega_{p i}$.

Let $a_{i}=2 \cos \left(\omega_{p i}\right)$, then $\omega_{p i}=\arccos \left(a_{i} / 2\right),-2 \leq a_{i} \leq 2$, $-\pi \leq \omega_{p i} \leq \pi$. The transfer function can be rewritten as

$$
H_{1}(z)=\frac{\prod_{i=1}^{k}\left(1-b_{i} z^{-1}+z^{-2}\right)}{\prod_{i=1}^{k}\left(1-r_{i} a_{i} z^{-1}+r_{i}^{2} z^{-2}\right)},
$$

where $b_{i}=2 \cos \left(\omega_{N i}\right), i=1,2, \ldots, k$.

For convenience, let $i=1$, then the notch frequency is $\omega_{N 1}$, pole radius is $r_{1}$, and (16) can be expressed as

$$
H_{1}(z)=\frac{B(z)}{A(z)}=\frac{1-b_{1} \cdot z^{-1}+z^{-2}}{1-a_{1} r_{1} z^{-1}+r_{1}^{2} z^{-2}} .
$$

Assuming that $\omega_{z}<\omega_{p}<\omega_{N 1}<\omega_{N 2}<\cdots<\omega_{N i}$, $\omega_{N 1} \geq \omega_{p}+B_{w} / 2$. With a given weighting function $W(\omega)$, we can define the cost function as

$$
C(a)=\int_{R} W(\omega)\left|H\left(e^{j \omega}\right)-H_{1}\left(e^{j \omega}\right)\right|^{2} d \omega,
$$

where the region $R=\left[\omega_{p}, \omega_{N 1}-\delta\right] \cup\left[\omega_{N 1}+\delta, \pi\right], \delta$ is a prescribed small positive number, combining (4) and (18) yields

$$
C(a)=\int_{R} W(\omega)\left|1-H_{1}\left(e^{j \omega}\right)\right|^{2} d \omega .
$$

To ensure the impacting characteristic of SIF and cancel the signal disturbing, the cascade notch filter should not affect the frequency response of the SIF; that is, the cost function should be minimized.

Substituting (17) into (19), we can obtain

$$
\begin{aligned}
C\left(a_{1}\right) & =\int_{R} W(\omega)\left|1-\frac{B\left(e^{j \omega}\right)}{A\left(e^{j \omega}\right)}\right|^{2} d \omega \\
& =\int_{R} \frac{W(\omega)}{\left|A\left(e^{j \omega}\right)\right|^{2}}\left|A\left(e^{j \omega}\right)-B\left(e^{j \omega}\right)\right|^{2} d \omega \\
& =\int_{R} \frac{W(\omega)}{\left|A\left(e^{j \omega}\right)\right|^{2}}\left|\left[\left(r_{1}^{2}-1\right) e^{-2 j \omega}+b_{1} e^{-j \omega}\right]-r_{1} a_{1} e^{-j \omega}\right|^{2} d \omega .
\end{aligned}
$$

Let $W(\omega) /\left|A\left(e^{j \omega}\right)\right|^{2}$ be the new weighting function, since $r_{1}$ and $b_{1}$ are known numbers, we need to find $a_{1}$ to minimize the cost function. For $a_{1}=2 \cos \left(\omega_{p 1}\right), \omega_{N 1}-\delta \leq \omega_{p 1} \leq$ $\omega_{N 1}+\delta$, we can use an iteration scheme to find the optimal value of $a_{1}$; each iteration refreshes the value of $A\left(e^{j \omega}\right)$. We

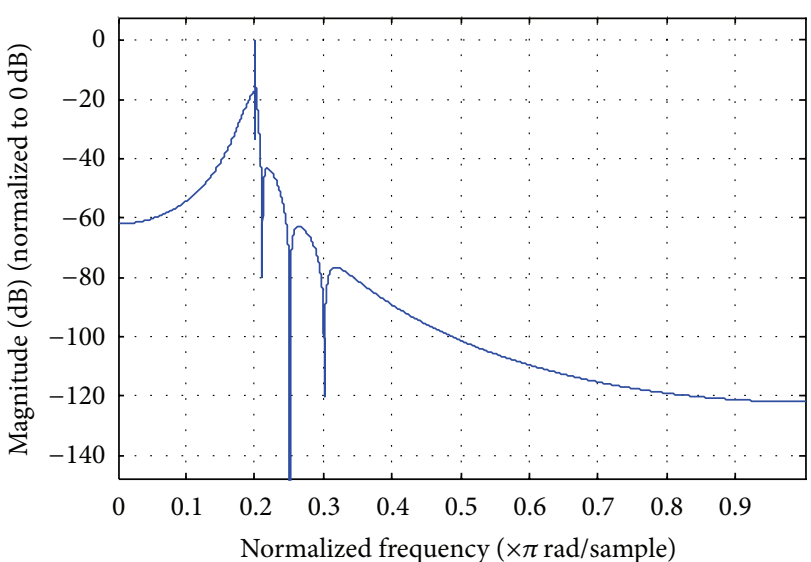

FIGURE 6: The frequency magnitude response of the designed CNSIF.

denote the value of $a_{1}$ at the $n$th iteration as $a_{1 n}, A\left(e^{j \omega}\right)$ as $A_{n}\left(e^{j \omega}\right)$, similarly. When $A\left(e^{j \omega}\right)$ gets the new value, the weighting function is updated immediately.

For convenience, substitute

$$
\begin{gathered}
p(\omega) \longleftarrow\left(r_{1}^{2}-1\right) e^{-2 j \omega}+b_{1} e^{-j \omega}, \quad q(\omega) \longleftarrow-r_{1} e^{-j \omega} \\
\Longrightarrow C\left(a_{1 n}\right)=\int_{R} \frac{W(\omega)}{\left|A_{n}\left(e^{j \omega}\right)\right|^{2}}\left|p(\omega)+q(\omega) a_{1 n}\right|^{2} d \omega \\
=\int_{R} \frac{W(\omega)}{\left|A_{n}\left(e^{j \omega}\right)\right|^{2}} \\
\cdot\left[a_{1 n}{ }^{2}|q(\omega)|^{2}+2 a_{1 n}|p(\omega) q(\omega)|\right. \\
\left.\quad+|p(\omega)|^{2}\right] d \omega \\
=\int_{R} \frac{W(\omega)}{\left|A_{n}\left(e^{j \omega}\right)\right|^{2}}\left[a_{1 n}{ }^{2}|q(\omega)|^{2}\right. \\
+2 a_{1 n} \cdot \operatorname{Re}\left(p(\omega) q^{*}(\omega)\right) \\
\left.+|p(\omega)|^{2}\right] d \omega .
\end{gathered}
$$

After some simplification, (21) is rewritten as

$$
\begin{gathered}
C\left(a_{1 n}\right)=\int_{R} W_{n}(\omega)\left[|q(\omega)|^{2} \cdot a_{1 n}{ }^{2}+2 \cdot \operatorname{Re}\left(p(\omega) q^{*}(\omega)\right)\right. \\
\left.\cdot a_{1 n}+|p(\omega)|^{2}\right] d \omega,
\end{gathered}
$$

where

$$
W_{n}(\omega)=\frac{W(\omega)}{\left|A_{n-1}\left(e^{j \omega}\right)\right|} .
$$




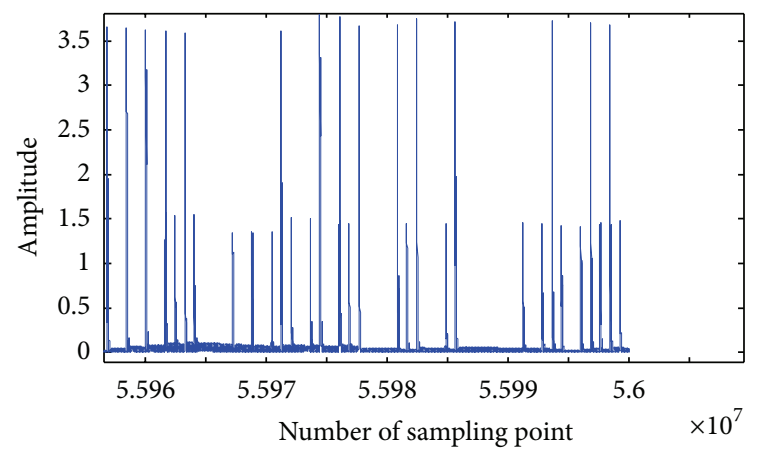

The last 20 output symbols of the subcarrier1: 00101110110100001011

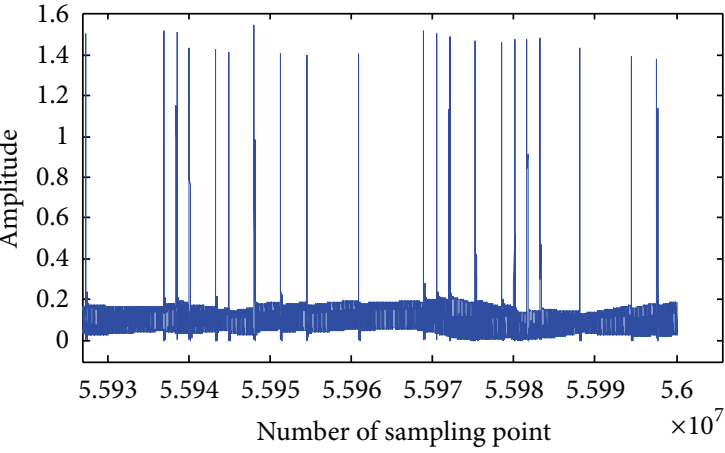

The last 20 output symbols of the subcarrier2: 11101011110010001010

FIGURE 7: The waveform after the CNSIF bank filtering.

So we can determine the value of $a_{1 n}$ by solving the constrained optimization problem

$$
\begin{aligned}
& \min \int_{R} W_{n}(\omega) d \omega \cdot[|q(\omega)|^{2} \cdot a_{1 n}{ }^{2}+2 \cdot \operatorname{Re}\left(p(\omega) q^{*}(\omega)\right) \\
&\left.\cdot a_{1 n}+|p(\omega)|^{2}\right]
\end{aligned}
$$

s.t. $\quad 0 \leq r_{1}<1$

$$
\begin{aligned}
& -2 \leq a_{1 n} \leq 2 \\
& \omega_{N 1}-\delta \leq \omega_{p 1} \leq \omega_{N 1}+\delta .
\end{aligned}
$$

We can see that the cost function is a parabola with its mouth opened up, so it could be transformed to a closed form and have an optimal solution. At the $n$th iteration, minimizing (22) subjected to constraints of (24) is a typical quadratic programming problem. We can compute the solution efficiently.

\section{Design Example}

5.1. Design Parameters. Set the normalized three notch frequencies as $0.21 \pi, 0.25 \pi$, and $0.3 \pi$. Easily derive the initial values that $a_{1}=0.521292, a_{2}=0.555360, a_{3}=0.587785$. Let $\delta=0.001 \pi$; the convergent values are $0.52206411,0.55613211$, and 0.58855711 . Given the following specifications: $r_{1}=r_{2}=$ $r_{3}=0.95$; weighting function $W(\omega)=1 ; \omega_{z}=0.6282 \mathrm{rad}$, $\omega_{p}=0.62855 \mathrm{rad}, B_{w}=\omega_{p}-\omega_{z}=0.00035 \mathrm{rad}$ $\left(1.114 \mathrm{e}^{-4} \pi \mathrm{rad}\right)$. The pole radii $r_{p}$ defined in (14) is 0.999999969375. Adding another three pairs of conjugate poles with angle $0.62855 \mathrm{rad}$, radii are $0.9,0.92$, and 0.95 , respectively. The frequency magnitude response of the final designed CNSIF is shown in Figure 6.

5.2. Performance. We choose the MCP-EBPSK signal as a source, simulating the performance of two subcarriers. The parameters are as follows: $f_{c 1}=10 \mathrm{MHz}, f_{c 2}=10.04 \mathrm{MHz}$, $f_{s 1}=160 \mathrm{MHz}, f_{s 2}=100.4 \mathrm{MHz}, A=1 ; B=1 ; \Delta=0.1 ; \eta=$ $0.5 ; K=2 ; N_{1}=100 ; N_{2}=160$; the entire test symbols are

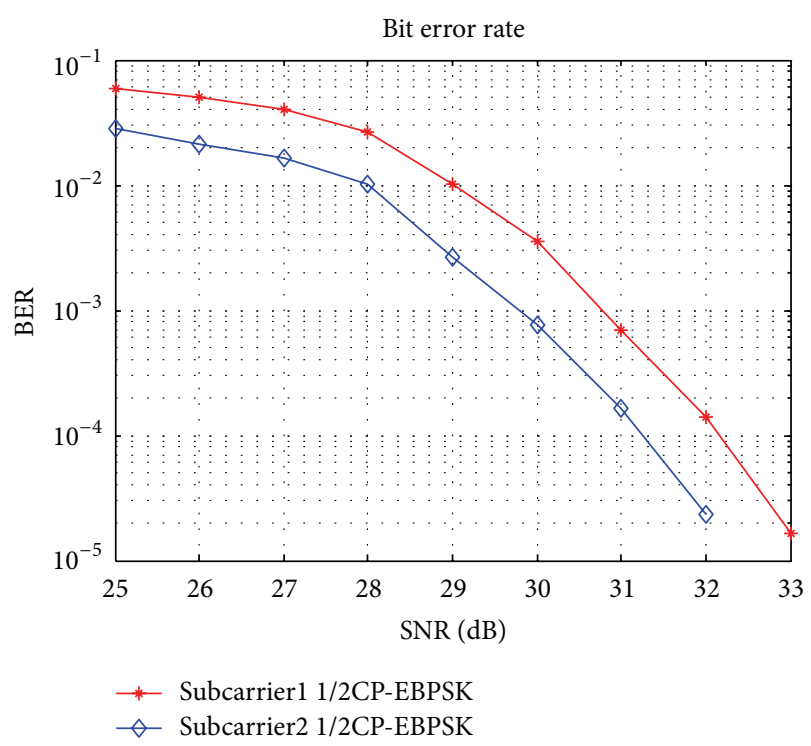

FIGURE 8: BER performances of two subcarriers.

3 million. Set the notch frequencies as $0.1255 \pi$ and $0.1992 \pi$, we can obtain $a_{1}=1.84656, a_{2}=1.62098$, after six iterations converge to 1.84855455 and 1.6219734 , respectively. Let $r_{1}=$ $r_{2}=0.95, \delta=0.001 \pi$, weighting function $W(\omega)=1$.

We can obtain the parameters of the CNSIF bank as

$$
\begin{aligned}
a_{1}=[1,-6.096111877753762,16.418167515832877, & -25.326641789930786,24.298560015038895, \\
& -14.721898165092053,5.447828484314318, \\
& -1.116449367584231,0.098333159057181] ; \\
b_{1}= & 0,0,0,0,1,-3.694313616799019,5.411987912102056, \\
& -3.694313616799019,1] ; \\
a_{2}= & {[1,-5.406525327518239,13.652579283244823,} \\
& -20.517799618098030,19.861352592576445, \\
& -12.536073668382514,4.996805854728122, \\
& -1.139889481405723,0.120047843082279] ;
\end{aligned}
$$

$b_{2}=[0,0,0,0,1,-3.239006288658281,4.622788277906668$, $-3.239006288658281,1]$. 
Figure 7 shows the waveform of the component signal after the CNSIF bank filtering. We can see that the designed CNSIF could separate the component MCP-EBPSK signals. The BER performance is shown in Figure 8.

\section{Conclusions}

With the intuitive method known as zero-pole placement, we analyzed the frequency response of the SIF in a quantitative way. The equation about the pole radii under specified bandwidth is derived. The designed CNSIF could not only maintain the impacting characteristic but also attenuate the interchannel interference (ICI). From Figure 6, we can also see that adding notch zeros could speed up the decaying of the response curve and tighten the bandwidth; this will help to filter the noise. Compared with OFDM, the multicarrier scheme of ABPSK could have quite low PAPR ratio. It can also be combined with MIMO, cognitive radio, and so forth to get better performance, which is our next work to emphasize.

\section{Acknowledgments}

The authors thank all of the reviewers for their valuable comments, which have considerably helped in improving the overall quality of the work presented in the revised paper. Editorial assistance and proofreading of the manuscript from Bingcheng Zhu are appreciated. This work is supported by the State 863 Project (2008AA01Z227), the National Natural Science Foundation of China (NSFC), under the Grant 61271204.

\section{References}

[1] H. R. Walker, "High Speed Binary Data Communication System [P]," US Patent 4742532, 1988.

[2] H. R. Walker, "VPSK and VMSK modulation transmit digital audio and video at $15 \mathrm{Bits} / \mathrm{Sec} / \mathrm{Hz}$," IEEE Transactions on Broadcasting, vol. 43, no. 1, pp. 96-103, 1997.

[3] H. R. Walker and B. Stryzak, "Comparing ultra wideband and ultra narrow band modulation," in Proceedings of the International Conference on Computing, Communications and Control Technologies (CCCT '04), Austin, Tex, USA, 2004.

[4] L. Wu, "The report of recent developments in UNB high-speed communications," Progress in Nature Science, vol. 17, no. 11, pp. 1467-1473, 2007.

[5] M. Feng, Research on key problems in high-efficiency modulation [Ph.D. thesis], Southeast University, Nanjing, China, 2008.

[6] F. He and L. Wu, "Analysis of power spectrum of continuous phase waveforms for binary modulation communications," in Proceedings of the International Conference on Ultra Modern Telecommunications and Workshops, Petersburg, Russia, October 2009.

[7] Y. Jin, L. Wu, A. Feng, and D. Lei, "An M-ary and continuous phase modem with random-polar," Chinese Journal of Radio Science, vol. 27, no. 5, pp. 931-936, 2012 (Chinese).

[8] T. A. Weiss and F. K. Jondral, "Spectrum pooling: an innovative strategy for the enhancement of spectrum efficiency," IEEE Communications Magazine, vol. 42, no. 3, pp. S8-S14, 2004.

[9] H. R. Walker, Ultra Narrow Band Modulation Textbook, 2010, http://www.vmsk.org/.
[10] M. Feng, P. Gao, and L. Wu, "Analysis and simulation of special filtering based on ultra narrow band modulated signal," Journal of Southeast University, vol. 40, no. 2, pp. 227-230, 2010 (Chinese).

[11] P. Gao, On impacting filter in unb receiver [M.S. thesis], Southeast University, Nanjing, China, 2010.

[12] J. A. Moorer, "The manifold joys of conformal mapping: applications to digital filtering in the studio," Journal of the Audio Engineering Society, vol. 31, no. 11, pp. 826-841, 1983.

[13] Z. Chen, L. Wu, and X. Chen, "The impacting filter bank design for MCP-EBPSK multicarrier demodulation," Signal Processing, vol. 28, no. 8, pp. 1063-1068, 2012 (Chinese). 


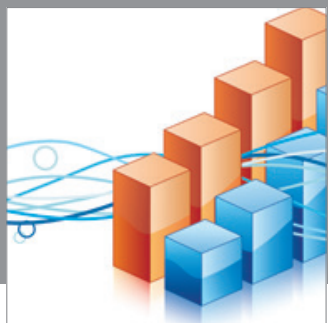

Advances in

Operations Research

mansans

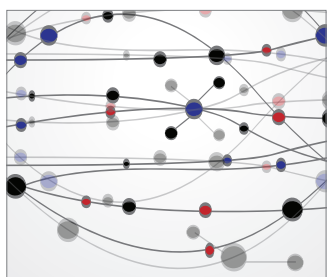

The Scientific World Journal
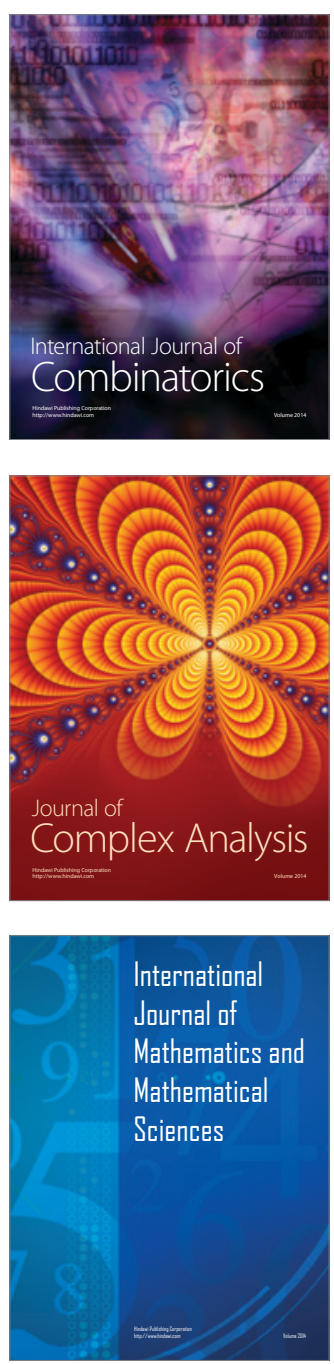
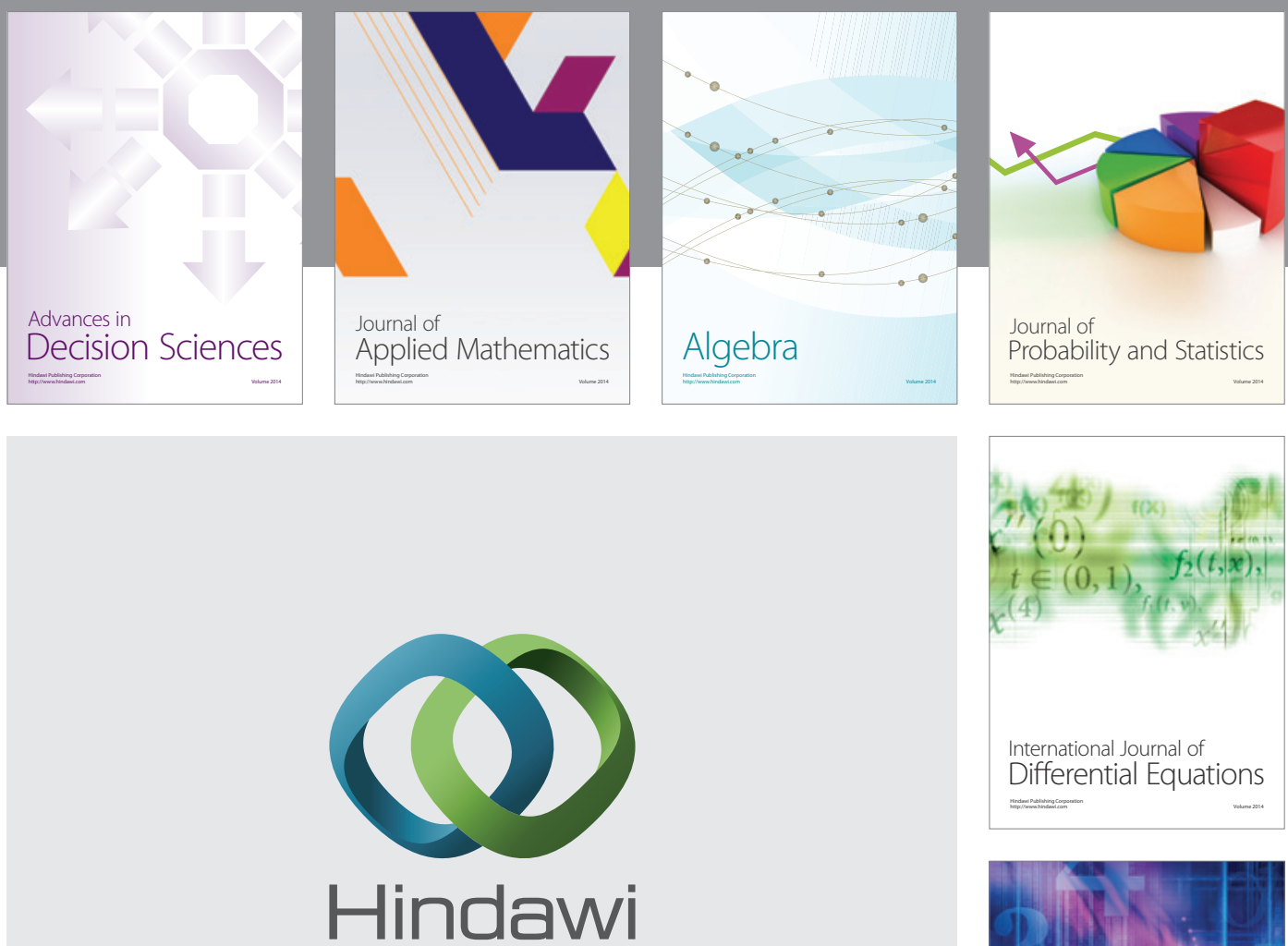

Submit your manuscripts at http://www.hindawi.com
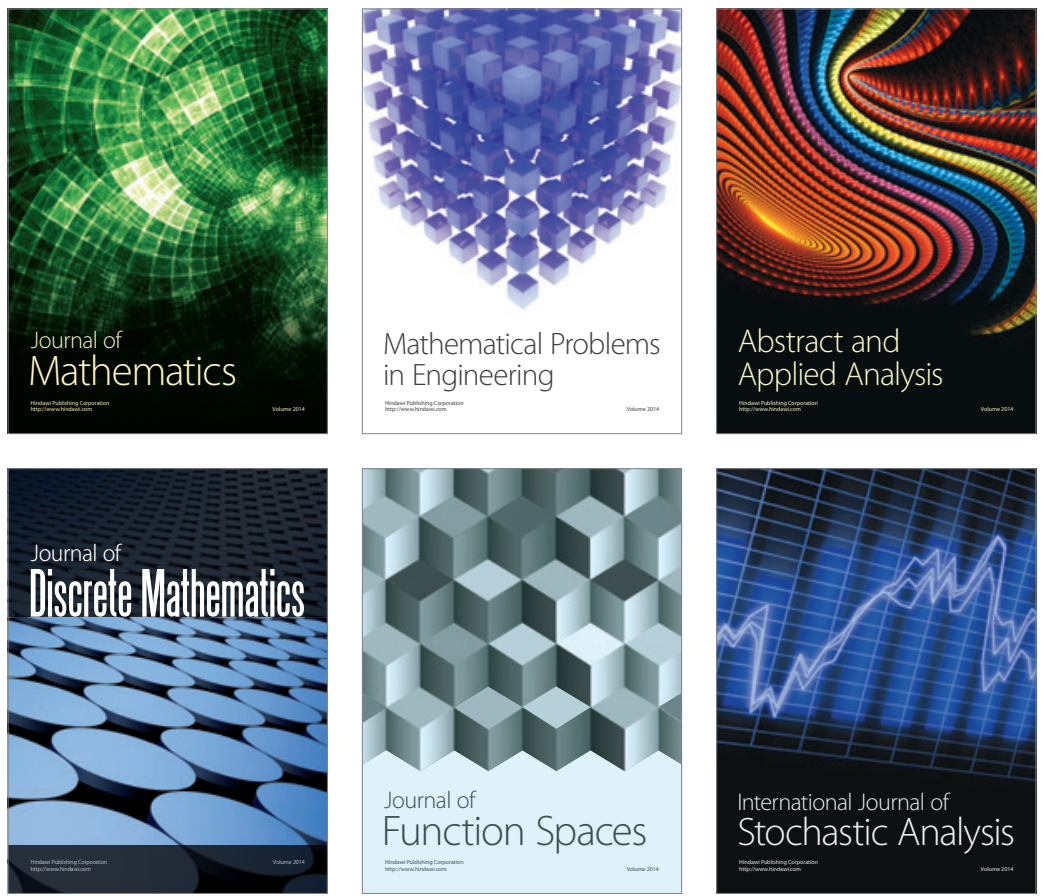

Journal of

Function Spaces

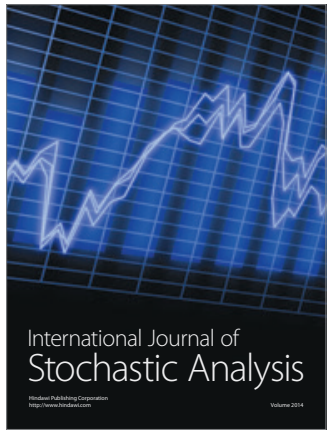

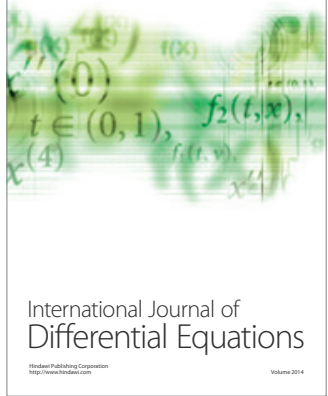
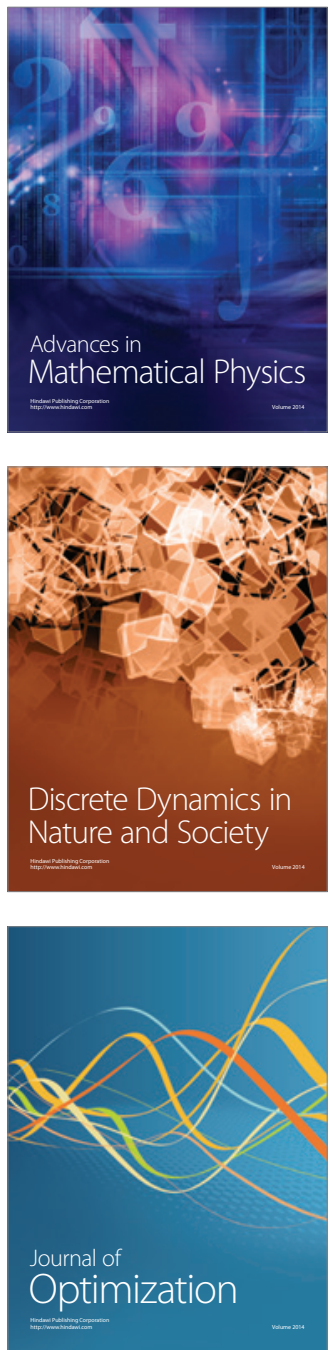